\title{
Patients with Mental Health History
}

By: Audrey Snyder and Julie Deters

Snyder, A. \& Deters, J. (2018). Patients with Mental Health History. In Martin, M., Heron, S. Walton, L.M. \& Strickland, M. (eds). Diversity and Inclusion in Quality Patient Care Case Compendium. Springer Publishing, Chapter 30, p. 219-224. https://doi.org/10.1007/978-3-31992762-6 30

***(C) 2019 Springer International Publishing AG. Reprinted with permission. No further reproduction is authorized without written permission from Springer. This version of the document is not the version of record and is subject to Springer accepted manuscript terms of use. $* * *$

\section{Abstract:}

This chapter contains a case study of a patient with mental health history who presents to urgent care for neck and back pain.

Keywords: Cushing's syndrome | bias | mental health | dorsocervical fat pad $\mid$ striae $\mid$ depression | back pain

\section{Book chapter:}

\section{Case Scenario}

A 43-year-old Caucasian female presents to the urgent care after hours clinic with a complaint of neck and midscapular back pain worsening over the last 2 days, which she rates as an $8 / 10$. She states she has had a lot of stress lately. She has had similar pain in the past with stress but not this bad. The pain is worse when she does anything over her head with her arms raised. The pain is not improved with heat, massage, or acetaminophen. She complains of generalized "muscle aches that have been going on for a while, but no one wants to address it." The patient is tearful as she describes what she calls her "struggles." She is dressed in a spaghetti-strap top and shorts. She is slightly diaphoretic. The outside temperature is in the high $80 \mathrm{~s}$. A neighbor brought her to the urgent care clinic. A nurse practitioner student assesses the patient first and reports her findings to the provider. The exam is remarkable for an obese Caucasian female with prominent buffalo hump, or dorsocervical fat pad, over lower cervical and upper thoracic posterior processes; diffuse striae over upper arms, legs, and abdomen; and galactorrhea. The remainder of the physical exam is unremarkable. The patient has a history of type II diabetes mellitus, which is uncontrolled. She was in the office last month for her annual exam with her primary care provider. When asked about the reported fasting blood glucose that was in the 300 s at her last visit, she states she has had trouble keeping a job because of depression and anxiety. She states the week this lab was drawn, she only had $\$ 5$ in her checking account and could not buy food, so she drank soda with sugar in it. She feels her anxiety is worse because of the unrelieved pain and financial concerns. 


\section{Review of Symptoms}

The patient reports she has trouble losing weight but does not think she has gained any recently. She reports difficulty walking due to generalized muscle pain. Depression is reported as baseline on current medications. Anxiety is worse as noted above. She denies difficulty swallowing, respiratory distress, and chest or abdominal pain. She reports galactorrhea, which has been persistent for several months. All other systems are reported negative.

\section{Past Medical History}

Depression, anxiety, schizoaffective disorder, type II diabetes uncontrolled, hyperlipidemia, metabolic syndrome, morbid obesity, post-traumatic stress disorder, sleep apnea, and vitamin D deficiency

Gynecologic history: P2, 1 live, 1 tubal, last pap 3 years ago, last mammogram 4 years ago

Surgical history: Left ulnar nerve transposition

Immunizations are up-to-date except for tetanus and diphtheria, which are 3 years overdue.

Medications Zocor $20 \mathrm{mg}$ daily at bedtime, aripiprazole $15 \mathrm{mg}$ daily at bedtime, metformin $\mathrm{Hcl}$ $1000 \mathrm{mg}$ extended release daily, fexofenadine $180 \mathrm{mg}$ daily, alprazolam $0.5 \mathrm{mg}$ daily as needed, chlorpheniramine maleate $4 \mathrm{mg}$ every 4-6 hours, Depakote ER $500 \mathrm{mg}, 2$ tablets at bedtime

Allergies Amoxicillin and penicillin

Health screenings Immunizations, tetanus 3 years overdue; last diabetic monofilament exam 11 months ago, due in 1 month; dilated eye exam 10 months past due; microalbumin due in 1 month; hemoglobin A1C (14\%) last month; lipid panel last month

Family History

Type II diabetes in paternal grandfather; no mental health concerns, hypertension, cancer, or other illnesses

Social History

Occupation: Not working currently, on unemployment

Marital status: Single

Children: One son

No history of tobacco, alcohol, marijuana, or illicit substance use reported.

Caffeine: coffee 3 servings per month, tea 1 serving per week, and soda 1-2 liters per day 
Health insurance: Currently has a policy with a high deductible

Physical Exam

Vital signs Temp, $98.7^{\circ} \mathrm{F}$; pulse, 69; BP, 100/66; respirations, 18; $\mathrm{O}_{2}$ sat $97 \%$ on room air; Ht 5 ft. 4 inches, weight 226 lb., BMI 38.8

General Obese Caucasian female appears stated age, appropriately dressed for weather, tearful and anxious but cooperative, good eye contact

HEENT Normocephalic, moon facies, CN II-X12 intact, trachea midline, no thyromegaly, nares clear, TMs with good light reflex bilaterally

Neck Supple, tender over lower cervical posterior processes, paraspinous muscle tenderness cervical and upper thoracic region, buffalo hump present, no adenopathy

Neuro Grossly intact, alert, and oriented x 3, intact memory for recent and remote events, upper extremities strength $4 / 5$, no obvious sign of deficit

Mental health/psych Depressed affect, expressed anxiety about current symptoms, denies suicidal thoughts, no agitation, normal thought processes

Cardiovascular S1S2 regular rate and rhythm, no murmurs, gallops, or rubs

Respiratory Bilateral breath sounds equal and clear

Abdomen Obese, round, soft, non-tender with normoactive bowel sounds all four quadrants, no hepatosplenomegaly

Extremities No cyanosis, no edema

Skin No rashes or lesions, reddish-purple striae over inferior aspect of upper arms, abdomen, and medial aspect of thighs

Breast No erythema, no palpable lumps, milky discharge from nipples bilaterally

The patient's medical record is reviewed for the past year. There is no record of the physical exam findings of dorsocervical fat pad, recent thyroid-stimulating hormone (TSH) lab, or endocrine workup. The patient denies being told about the fat pad on her back. She is prescribed cyclobenzaprine $10 \mathrm{mg}$ every $8 \mathrm{~h}$ as needed to help her muscles relax and placed on ibuprofen $600 \mathrm{mg}$ every 6-8 hours for 2 days and then as needed for pain. TSH and adrenocorticotropic hormone (ACTH) levels and a 24-h urine free cortisol level are ordered. The patient is scheduled for a follow-up visit in 2 weeks with her primary care provider. The patient's primary provider is sent an electronic message through the medical record indicating the abnormal findings and expressing concern for Cushing's syndrome, as well as mentioning scheduling an appointment 
and the possible need for MRI and endocrine referral. The primary provider responds, "You know she drinks sodas every day. She has lots of complaints that have no basis. She frequently cancels her appointments and will probably be a no show" [1].

\section{Questions for Discussion}

1. What was said by whom, to whom?

The patient said, "Muscle aches that have been going on for a while but no one wants to address it."

Attitudes/Assumptions: The Patient

(a) My high deductible insurance plan prevents me seeing a doctor when I am sick until it is really bad.

(b) Doctors just think my physical complaints are all in my head.

The doctor wrote, "You know she drinks sodas every day. She is a very noncompliant patient. She has lots of complaints that have no basis. She frequently cancels her appointments and will probably be a no show."

Attitudes/Assumptions: The Primary Care Provider

(a) This patient is not adherent to an ADA diet or recommendations.

(b) This patient has a psychiatric history and creates complaints.

(c) This patient is not working and is probably drug seeking with the complaint of "back pain."

Gaps in Provider Knowledge

(a) Lack of awareness of Cushing's syndrome constellation of symptoms.

(b) Lack of knowledge of patient's financial situation impacting food choices and ability to keep appointments in clinic. Assess patient reasons for nonadherence versus labeling patient as noncompliant.

(c) Lack of knowledge of disparities/discrimination: Persons with a medical history of mental health diagnoses are often subject to bias [2].

2. What was done?

Urgent care provider identified that patient has a conglomeration of symptoms that may reflect Cushing's syndrome. She ordered the appropriate lab test. She followed up with the patient on lab results, encouraged her to keep the primary care provider follow-up appointment, and asked about the patient's ability to obtain a ride to the clinic.

3. What actions could have been taken by the primary care provider for earlier identification of a potential problem? 
(a) Perform a comprehensive physical exam every year.

(b) Assume a patient with a mental illness who presents with medical complaints is ill.

(c) Do not assume that all patients who present with back pain are drug seeking [3].

(d) Check the Controlled Substance Prescription Monitoring Program online to see if the patient is receiving narcotics from other sources.

4. What medical issues concern you about this case?

(a) The patient has significant persistent physical findings for Cushing's syndrome that have not been previously documented or evaluated. Signs and symptoms of Cushing's syndrome include truncal obesity, moon face, hypertension, skin atrophy and bruising, diabetes or glucose intolerance, gonadal dysfunction, muscle weakness, hirsutism/acne, mood disorders, osteoporosis, edema, polydipsia/polyuria, and fungal infections. Truncal obesity is the most common manifestation [4]. Clinical suspicion of Cushing's syndrome "arises in the presence of central obesity with face and supraclavicular fat accumulation, a cervical fat pad, thinned skin, purple striae, proximal muscle weakness, fatigue, hypertension, impaired glucose metabolism and diabetes, acne, hirsutism, and menstrual irregularities" [4].

(b) In a fast-paced primary care environment, patients may not be fully undressed for physical exams.

(c) Social circumstances should be evaluated with patient encounters. Ancillary staff may be helpful in updating this information.

\section{Pearls and Pitfalls}

Pearl The urgent care provider completed a comprehensive history and physical exam, not just a problem-focused exam, when the patient presented in the urgent care environment. A detailed social history was also obtained.

Pearl The urgent care provider listened to the patient's concerns even though the patient felt previous providers were not listening. Mental health history was not the focus of the chief complaint.

Pitfall This patient could be lost to follow-up since she presented in an urgent care environment.

\section{Case Outcome}

Diagnosis Muscle spasms in neck and back, concern for Cushing's syndrome, depression, anxiety, type II diabetes mellitus

Disposition The patient returned to the primary care clinic for her follow-up appointment. Her pain was tolerable at a rating 3-4/10 while on muscle relaxants and NSAIDS. TSH was elevated at 6.5 , and she was started on levothyroxine. ACTH level was assessed as intermediate at 13. A MRI of the pituitary was ordered, and the patient was referred to endocrinology. She expressed gratitude for her symptoms being assessed and addressed. 


\section{References}

1. Keeling A, Utz SW, Shuster GF 3rd, Boyle A. Noncompliance revisited: a disciplinary perspective of a nursing diagnosis. Nurs Diagn. 1993;4(3):91-8.

2. Kaplan A. Bias against schizophrenic patients seeking medical care [Internet]. [place unknown]: Psychiatric Times; 2013 [cited 2017 Nov 6]. Available from:

http://www.psychiatrictimes.com/apa2013/bias-against-schizophrenic-patients-seekingmedical-care.

3. Grover CA, Eder JW, Close RJH, Curry SM. How frequently are "classic" drug-seeking behaviors used by drug-seeking patients in the emergency department? West J Emerg Med. 2012;13(5):416-21.

4. Boscaro M, Arnaldi G. Approach to the patient with possible Cushing's syndrome. J Clin Endocrinol Metab. 2009;94(9):3121-31. 